\title{
Basura: Procesos de trabajo e impactos en el medio ambiente urbano
}

\author{
Héctor Castillo, Margarita Camareria y Alicia Ziccardi*
}

El articulo tiene como objetivo presentar una visión global de los diferentes componentes técnicos, económicos y sociales que están presentes en la organización de las tareas de recolección y disposición final de la basura; a partir de ella se extraen algunos elementos que sirven para la evaluación de sus efectos sobre el medio ambiente.

Para ello se señalan algunos lineamientos y orientaciones teóricas sobre la relación entre la basura y el medio ambiente, y también se analiza la planeación ambiental por parte de las instituciones gubernamentales. Posteriormente se presenta la perspectiva so. cial y urbana tomando los casos de la ciudad de México y de Ciudad Juárez, espacios urbanos en los que se analizan las diferentes formas de organización técnica y social del trabajo que prevalece en los tiraderos. Finalmente, se expresan algunas ideas acerca de los factores que deben contemplarse para proponer otras formas de eliminación y de aprovechamiento de los desechos sólidos a fin de garantizar una mayor calidad del medio am. biente urbano.

\section{Introducción}

Los problemas generados por las modalidades que presenta el proceso de recolección y disposición de basura en las grandes ciudades del país constituyen una cuestión insoslayable para la temática ambiental. Contaminación del suelo y la atmósfera, insalubridad en los tiraderos y sus alrededores, deterioro ambiental del espacio urbano, son manifestaciones visibles de las deficiencias que caracterizan al sistema de recolección de residuos. La forma como la sociedad desecha sus residuos ejerce marcados efectos sobre la calidad de vida del conjunto de la población que habita determinado espacio urbano. Detrás de dichos efectos existe un servicio público de recolección de desechos más o menos organizado, que tiene como destino final de los mismos la existencia de un particular universo económico, social y político: el tiradero a cielo abierto.

El tipo de relaciones sociales que se generan en el interior del tiradero, las peculiaridades de la mano de obra que se integra al proceso de trabajo de selección y clasificación de residuos, las prácticas de organización del trabajo que se desarrollan en el momento de la recolección y del aprovechamiento de la basura, los mecanismos socio-políticos generados en torno a esta actividad (representando intereses particulares), las formas de actuación de las institu-

* Instituto de? Investigaciones Sociales, Lnversidad Nacional Automoma de: Me viee 
ciones gubernamentales responsables, ante la ciudadanía, de la eficacia de este servicio, constituyen suficientes elementos para evaluar la complejidad que encierra la problemática que nos ocupa. Por ello, pensar en los problemas ambientales negativos que surgen por la existencia de los tiraderos a cielo abierto en el medio urbano, implica necesariamente introducirse en este universo de análisis sociológico específico, ya que para intentar modificar los efectos nocivos al ambiente sería necesario alterar las relaciones económicas sociales y políticas que convergen actualmente tanto en la recolección como en la disposición final de la basura de la sociedad.

En este sentido, el principal objetivo que se persigue al presentar este breve artículo es el de ofrecer al lector ocasional una visión global de los diferentes componentes técnicos, económicos y sociales que están presentes actualmente en los procesos de trabajo relacionados con las tareas de recolección y disposición final de la basura, para de ahí ofrecer algunos elementos que sirvan para la evaluación de los efectos que dicha organización tiene en el medio ambiente, así como mostrar la necesidad que se tiene de observarlos unificadamente, ya que, en buena medida, la existencia de unos repercute de una u otra forma en los otros.

Para ello se ha organizado el trabajo respondiendo a dos interrogantes principales: ¿Cómo se organiza actualmente -técnica, económica y socialmente- el sistema de recolección y disposición de basura? y, ¿de qué manera influye este tipo específico de organización en el medio ambiente?

En la primera parte se indican a grandes rasgos algunos lineamientos y orientaciones teóricas que intentan reflexiones sobre la relación entre la basura y el medio ambiente, o sea, la forma como la investigación social ha abordado la temática, además de incluir algunas notas importantes sobre la evaluación y propuesta que encierra la planeación ambiental por parte de las instituciones gubernamentales en torno a esta cuestión. Seguidamente se presenta la perspectiva social y urbana de análisis, señalando los dos casos estudiados específicamente: Ciudad de México y Ciudad Juárez, que serán precisamente los espacios urbanos en los que se analizarán las diferentes formas de organización técnico-social del trabajo que prevalece en los tiraderos. La tercera parte del trabajo sintetiza algunas de las principales técnicas que se desarrollan actualmente para el control de la contaminación provocada por los desechos sólidos, enfatizando sus ventajas y/o desventajas de acuerdo al tipo de desechos que se producen en México. Finalmente, se presentan algunas consideraciones generales para explicar el peso real que tienen los distintos grupos de pepenadores de los tiraderos y el freno que 
representan para modificar los sistemas actuales de disposición de desechos (que afectan directamente al medio ambiente), para dejar señaladas algunas proposiciones que pudieran servir posteriormente para garantizar una mejor calidad del medio ambiente urbano, en general, y del medio de vida de los trabajadores que desempeñan estas actividades, en particular.

\section{Basura y medio ambiente}

Las relaciones existentes entre la forma como se efectúa en la sociedad el servicio de recolección, reaprovechamiento y/o eliminación de residuos y el impacto ambiental que tal proceso genera demanda la atención tan to de investigadores como de representantes de las instituciones socialmente responsables de ofrecer este servicio a la comunidad. En este sentido una primera aproximación a la cuestión contenida en planteamientos teóricos generales, así como los propios resultados de las investigaciones emprendidas y los lincamientos de la planeación y acción gubernamental, permiten enmarcar la problemática de la basura y el medio ambiente.

En el más elevado nivel de abstracción se han explorado los problemas de desajuste que presenta la modalidad de articulación sociedad-naturaleza. Entre ellos se ha señalado que la acumulación de desechos junto con el uso y explotación in adecuada de los recursos naturales y la modificación de los espacios naturales por la implantación de sistemas urbanos industriales, constituyen sintomas que poseen "un efecto significativo sobre el ser humano, a través de impactos directos o indirectos sobre la salud y la calidad de vida, así como sobre el proceso de desarrollo económico social..." (Sánchez, Vicente (a), 1983, p. 6). De esta forma se enfatiza el hecho de que la acumulación de desechos condiciona diversos tipos de contaminación "sea porque sobrepasa en el tiempo la capacidad de la naturaleza para metabolizarlos y reciclarlos, sea porque produce desechos no reciclables" (Idem, p. 5). De esta forma la sociedad ejerce presiones sobre el sistema natural y es necesario hallar opciones que restablezcan una adecuada interacción entre las acciones del sistema social y del sistema natural (Sánchez (a), Vicente, 1983). Es precisamente la preocupación que encierra esta búsqueda de una nueva "racionalidad" que garantice una apropiación de la naturaleza para obtener el crecimiento económico deseado pero sin provocar efectos negativos sobre el medio ambiente, el principal elemento que estructura a esta corriente del pensamiento sobre la cuestión am- 
biental. ${ }^{1}$ Se señala también que el problema no encuentra en opciones tecnológicas "apropiadas" el único elemento en el cual fundar una racionalidad productiva diferente a la racionalidad tecnológica del capital, que ha sobreexplotado los recursos naturales y humanos en diversas regiones, provocando graves problemas ambientales (Leff, Enrique, 1984, p. 75). Particularmente en los países en vías de desarrollo las formas de organización de la producción basadas en actividades humanas que permitan aprovechar elementos y utilizar desperdicios con fines productivos, han sido consideradas como características fundamentales en la construcción del concepto de ecodesarrollo. ${ }^{2}$

Por otro lado, no sólo se trata de considerar las formas organizativas de la fuerza de trabajo como alternativas que debe estructurar el capital en la búsqueda de excedentes, sino que debe considerarse el conjunto de componentes sociales que están presentes en el proceso productivo. Sólo así podrá reorientarse la acción sobre la naturaleza: "los problemas relativos al medio natural generados por la manera como los hombres desarrollan sus actividades son también y quizá fundamentalmente, problemas sociales. Es decir, que los aspectos técnicos son solamente una dimensión de los mismos, aunque una dimensión de considerable importancia" (Pires, Pedro, 1982, p. 21).

En estas teorizaciones podemos hallar importantes sugerencias para analizar el problema que nos ocupa. En lo fundamental, surge la idea de que la búsqueda de alternativas productivas que provoquen menores impactos ambientales no puede centrarse únicamente en hallazgos tecnológicos. Particularmente en nuestros países la abundancia de mano de obra exige pensar en formas de organización del trabajo que incorporen fuerza de trabajo masivamente, a la vez que garanticen una mayor igualdad social. El universo del trabajo manual de los tiraderos de la ciudad de México constituye, sin temor a equivocarnos, un espacio en el que se estructuran relaciones sociales expoliativas para esa mano de obra que procura una actividad remunerada en el medio urbano y además un lugar donde habitar. Introducir importantes cambios tecnológicos en esos tiraderos produciría efectos drásticos en los miles de pepenadores que viven y trabajan en los tiraderos, de ahí su oposición permanente a los cambios y modificaciones que han propuesto "modernizar" o "tecnificar" el destino final de los desechos, los cuales, hasta la fecha, no han podido alterar los mecanismos de explotación que pre-

\footnotetext{
Cf. Sachs, Ignacy, 1982, pp. 74 y 75

${ }^{2}$ Cf. Idem, pp. 40 y 41.
} 
valecen en su interior. Formas alternativas de organización social de este trabajo - p. ej. cooperativas de pepenadores - ofrecen ciertamente mayores posibilidades de que esta actividad constituya una opción laboral valorada socialmente de manera positiva y sientan las bases para concretizar una política de control sobre los efectos ambientales que provocan los tiraderos.

Cualquier planteamiento sobre la manera como puede controlarse el impacto ambiental de la basura deberá considerar la realidad social particular que se genera alrededor de estas actividades. Muy difícilmente en un universo en el que prevalece el caciquismo y los más elevados grados de explotación de la fuerza de trabajo puede pensarse en introducir mecanismos técnicos y/o sociales que tengan en cuenta los intereses comunitarios, tal como es mejorar la calidad en ambiente. La situación económica y política que caracteriza a esta actividad en esos tiraderos no puede articularse a la defensa de intereses colectivos. Frente a ello, la investigación social ha avanzado desde la perspectiva de señalar cuáles son las condiciones técnico-naturales que caracterizan al problema de la basura en relación con su impacto ambiental. Así, se ha señalado que "la disposición de desechos sólidos representa un problema doble y un tanto irónico para el D.F.: tales desechos contienen recursos básicos como el papel, el vidrio, el plástico y materiales orgánicos; son productos que se pueden reutilizar con gran ventaja, pero cuando se tiran crean problemas de disposición final, salud pública y contaminación. El análisis de la composición material de los desechos sólidos es importante para reconocer y cuantificar tanto los daños ambientales y la pérdida de recursos, como los niveles probables de utilización de ciertos materiales, en caso de que se instituyan programas al respecto" (Restrepo, Ivan y Phillips, David, 1982, p. 22).

Este tipo de análisis avanza en el sentido de considerar que mientras una parte de los desechos son reutilizables y se transforman en mercancías, otros deben ser eliminados. La presencia de fuertes intereses económicos individuales en los tiraderos de la mayoria de las ciudades, establece marcados límites a este trabajo de selección de materiales que emprenden los trabajadores. Si desde un punto de vista social conviene reutilizar la mayor cantidad de desechos sólidos posible, ello sólo será realizable (por el momento al menos) en tanto el trabajo que implica esta reutilización no altere las formas de obtención de ganancias que posee el o los concesionarios que controlan el trabajo de selección de materiales al interior de los tiraderos.

Por otra parte, la cuestión de responsabilidad social indica que los tiraderos a cielo abierto constituyen graves focos de contamina- 
ción ambiental a la vista de cualquier ciudadano. La gravedad de los problemas que generan no puede ser más claramente descrita que como lo hace el siguiente texto: "Los tiraderos de basura a cielo abierto contaminan el suelo por los productos químicos que resultan de la putrefacción o fermentación de la basura; si se les quema contaminan la atmósfera y provocan problemas de olores nauseabundos en los alrededores. Este tipo de contaminación atrae o incrementa la fauna nociva como las ratas, las moscas y otros insectos y gusanos maléficos que viven y se reproducen en grandes cantidades; propicia la insalubridad y las enfermedades transmisibles ya que el viento reparte gran cantidad de desechos en enormes extensiones, deteriorando el ambiente natural. En general, los tiraderos hacen deprimente el paisaje y contribuyen a disminuir la calidad de vida por sus características negativas en cuanto a aspectos de higiene, salud y bienestar público" (Trueba, José, 1980, p. 111).

Por ello el poder público y la sociedad en su conjunto no pueden permanecer ajenos. Existen propuestas de introducción de tecnologías apropiadas que podrían contribuir a disminuir la cantidad de desechos que genera la población a partir de la introducción de sistemas relativamente simples de reciclaje de desechos. Entre éstos, se encuentran las propuestas para involucrar a los usuarios en la clasificación de sus desechos sólidos a escala domiciliaria, que a pesar de que ya se aplican con éxito en diversas ciudades (principalmente de países industrializados) en el caso de México son prácticamente nulos, o bien, sólo han quedado en buenas intenciones por parte de las autoridades que los han promocionado en algunas contadas ocasiones. $^{3}$ Por otra parte, en el área de los desechos orgánicos, el SIRDO (Sistema Integral de Reciclaje de Desechos Orgánicos) desarrollado por el Grupo de Tecnología Alternativa A.C. constituye una propuesta de tecnologías de bajo costo y fácil manejo mediante las cuales la comunidad puede obtener abono rico en nutrientes a partir de incorporar la basura orgánica a las cámaras húmedas en donde se descompone junto con la excreta.

Pero ciertamente el problema de la basura es un problema social generado principalmente por las actividades urbano-industriales y, como tal, cabe al Estado participar activamente en la solución del mismo. En este sentido la planeación ambiental instaurada en el presente sexenio al más elevado rango gubernamental con la creación de una Subsecretaría de Ecología, es la encargada de introducir la cuestión del medio ambiente y la basura entre sus planteamientos en torno a la contaminación. En un diagnóstico que intenta detec-

3 Cf. Fortalecimiento y Desarrollo Municipal, 1983. 
tar las causas que han provocado el deterioro ambiental, particularmente en la ciudad de México, se considera que las modalidades que ha asumido el proceso de industrialización que ha protagonizado el país han traído consigo un significativo deterioro del medio ambiente, y que la acción gubernamental hasta entonces no ha actuado para reorientar el mismo. Se explícita que: "Este proceso estaba sustentado en un conjunto de políticas que favorecian al desarrollo de la industria entre las que destacan el otorgamiento de insumos básicos subsidiados como la energía y agua, la ausencia de control sobre la degradación del medio ambiente y la generación de desechos y residuos: esto es por las emisiones de humos, polvos y gases contaminantes, de aguas residuales, basura y otros" SPP, (Plan Nacional de Desarrollo, 1982-1988, p. 246).

La concentración industrial en el área metropolitana de la ciudad de México, considerada por el diagnóstico oficial como una de las cinco ciudades del mundo más contaminadas, constituye una de las principales causas de la situación que prevalece. Pero como es sabido, el transporte es la principal fuente de contaminación atmosférica. La basura, la generación de desechos sólidos, contribuye ciertamente agravando aún más una crítica situación y ello es consecuencia de "una carencia de adecuados sistemas de recolección, tratamiento y disposición final de desechos sólidos, acordes a la realidad del país en sus diferentes regiones. La modificación de sus patrones de consumo ha aumentado la generación per cápita de basura, incrementándose el volumen de materiales no biodegradables y desechos industrialmente tóxicos. Desafortunadamente, nunca se ha reglamentado el manejo de estos residuos y ante el incremento demográfico e industrial, en una sociedad orientada hacia el consumo, éste ha sobrepasado la capacidad de acción de las autoridades locales" (SPP, Plan Nacional de Desarrollo, 1983-1988, p. 248).

Lo que este diagnóstico enfatiza es la necesidad de normar las funciones que deben cumplir las instituciones del sector público, particularmente los gobiernos municipales, y cuál es la responsabilidad del sector privado, particularmente del capital industrial. Esto parece importante en tanto no existe una legislación clara al respecto. Pero ciertamente, ello no basta para abordar el problema de los desechos sólidos que se generan en las ciudades del país; por ello se enuncia la intención oficial de elaborar una estrategia que contemple criterios tales como el tamaño de las ciudades, la estructura de las mismas, el número de habitantes y la topografia, entre otros factores. ${ }^{4}$ Como se verá a continuación, la existencia de los problemas

${ }^{4}$ Cf. Plan Nacional de Desarrollo, 1983-1988, p. 242. 
remite a causas técnicas, económicas y políticas más profundas, las cuales necesariamente deben ser consideradas para elaborar una acción que efectivamente modifique el estado actual del problema. Las tres líneas de acción que enuncian los organismos planificadores se inscriben en la elaboración de una política de corte normativo, la cual puede generar opciones consensuales favorables pero es distante de la política efectivamente implementada. Estas líneas son: 1) racionalizar la generación y reciclaje de residuos sólidos; 2) mejorar la calidad y cobertura de los servicios de recolección, tratamiento y disposición final, y 3) establecer proyectos de capacitación, de difusión y educación encaminados a fomentar y establecer la conciencia ecológica y una amplia participación ciudadana. Desde una perspectiva técnica se explícita la necesidad de "establecer sistemas de recolección, manejo y disposición final de residuos sólidos que incluyen el establecimiento de rellenos sanitarios y cementerios industriales". ${ }^{5}$

Todos estos elementos indican la existencia de una preocupación institucional del gobierno federal sobre una problemática que necesariamente debe estar incorporada al conjunto de políticas que el Estado está elaborando en materia de preservación del medio ambiente. En el caso de la política del gobierno de la ciudad de México, existe la intención de enfrentar el complejo problema de generación de las 14000 ton en promedio de desechos sólidos diarios (considerando tanto el Distrito Federal como su área metropolitana de los que cerca de $75 \%-10500$ ton - se depositan en los tiraderos a cielo abierto), a partir de "modificar los patrones urbanos de generación de desperdicios y someter a tratamiento e incineración a la basura". ${ }^{6}$ Concretamente, y como se verá más adelante, se trata de optar por la instalación de una planta incineradora de residuos lo cual, si bien ataca el problema de la acumulación de residuos en la ciudad, genera otros impactos ambientales negativos como consecuencia de los procedimientos en que se basa dicha eliminación.

Estas primeras aproximaciones al estudio de la relación basura. medio ambiente indican que se trata de una cuestión extremada. mente importante en la cual, diferentes agentes sociales, (ciudada.

5 Cf. SPP, Programa Nacional de Ecología, p. 58. En atención al suelo agrícola el Programa establece que "se controlará el uso y manejo indiscriminado de residuos y envases de fertilizantes y pesticidas, entre otros, mediante el establecimiento de centros receptores de agroquímicos...", 1983.

${ }^{6}$ Cf: Departamento del Distrito Federal-Secretaría General de Desarrollo Urbano y Ecologia, "Programa de reordenación urbana y protección ecológica del D.F." México, D.F., 1984. 
nos, instituciones gubernamentales, investigadores, técnicos, etc.) deberán realizar un esfuerzo por mejorar y reorganizar la situación que actualmente prevalece.

\section{Perspectiva del análisis}

Se ha enfatizado ya la complejidad que encierra en sí mismo el análisis del proceso de recolección y disposición final de la basura en el medio urbano. Repensar los resultados de investigación de que se parte, introduciendo ahora la dimensión ambiental del problema, constituye un nuevo desafio. ${ }^{7}$ Para ello, esta parte del trabajo se abocará inicialmente a presentar un conjunto de señalamientos generales surgidos de la observación de los procesos en torno al problema de la relación basura-medio ambiente. Los casos que servirán de apoyo a esta reflexión serán la ciudad de México y Ciudad Juárez, Chihuahua, escenarios francamente diferentes tanto desde la perspectiva del tipo y magnitud de las actividades económicas que se despliegan en ambos espacios urbanos, como de la fuerza de trabajo que los habita y del sector que se inserta en las actividades de disposición final de la basura, las formas de organización técnicosocial del trabajo que se desarrollan en los tiraderos de cada ciudad y los efectos ambientales que pueden derivarse de situaciones marcadamente diferenciales.

Analíticamente distinguiremos dos aspectos fundamentales del proceso de recolección y disposición de basura:

1) Un aspecto técnico: ligado a los procesos de organización técnico-social del trabajo a partir del cual se transforman los desechos sólidos que llegan al tiradero: reutilización y/o eliminación de la basura, previa selección.

2) Un aspecto social: referido a las formas de incorporación de la mano de obra (seleccionadores, segregadores o pepenadores) al proceso de trabajo que se ejerce en los tiraderos; las condiciones de vida de estos trabajadores así como la retribución económica que obtienen, las prácticas sociales y políticas a partir de las cuales se estructuran relaciones sociales particularmente entre los agentes involucrados (concesionarios, fuerza de trabajo, instituciones gubernamentales, organizaciones de la sociedad civil, etcétera).

Se parte de la idea de que la temática de la basura y el medio ambiente alude a un fenómeno en el que las posibilidades de imponer una racionalidad alternativa a la que prevalece actualmente, descansa en otorgar al componente social (concretamente a la creación

${ }^{7}$ Castillo, Héctor, 1983 y Castillo, H., Camarena, M. y Ziccardi, A. 1985. 
de formas de organización social del trabajo alternativas a las existentes), una importancia, si no única, sí central. Tal como se verá, existen opciones tecnológicas que pueden contribuir a mejorar la calidad del servicio público de recolección de basura y del destino final de los desechos, aunque debe resaltarse que en tanto se trata de maquinarias y equipos fundamentalmente de importación son obvias las limitaciones que estas opciones encierran en la coyuntura actual de crisis económica por la que atraviesa el país. Por otra parte, con ello no asegura una disminución de los efectos ambientales negativos generados por la existencia de tiraderos a cielo abierto aun cuando se apliquen diferentes técnicas de relleno sanitario, entre otras cosas, dado que existen "derechos otorgados" hacia los pepenadores y sus distintas organizaciones desde hace ya varias décadas en los cuales está contemplada implícitamente la idea de que "la basura es de los pepenadores que la trabajan y como tal debe de respetarse ese desecho" a decir de diversos funcionarios del Departamento del Distrito Federal (DDF) y del municipio de Ciudad Juárez.

Por esto, cualquiera de estas opciones debe ser analizada necesariamente desde una perspectiva social global a fin de poder encarar el problema desde sus causas. Parece poco probable pensar en la utilidad práctica de una evaluación ambiental del impacto de tal o cual tiradero o el servicio de limpia en su conjunto desde una perspectiva meramente cuantitativa. Cualquier medición de contaminación no será suficiente para elaborar una propuesta alternativa que contribuya a mejorar la calidad del medio ambiente. En todo caso ofrecerá un diagnóstico más preciso del deterioro alcanzado, pero la solución del problema descansa necesariamente en la comprensión cualitativa del mismo.

A continuación se presentan de manera resumida algunos de los datos más sobresalientes de los dos estudios de caso señalados, con el fin de profundizar un poco más en las características particulares de la organización de los procesos de trabajo en cada uno de ellos.

\subsection{El caso de la ciudad de México}

La basura de la ciudad de México presenta un caso de estudio que puede ser comparable (desde el punto de vista ecológico) al de cualquier otra ciudad de América Latina que tenga como sitio para la disposición final de sus desechos los "tiraderos a cielo abierto". En estas enormes extensiones de terreno se va acumulando la basura 
de manera indiscriminada, materia orgánica e inorgánica, que entra en estado de descomposición y putrefacción y que provoca serias consecuencias ambientales a distintos niveles: 1) en el agua y suelo, por las filtraciones de sustancias tóxicas que llegan incluso muchas veces a los mantos acuíferos y por la erosión que provocan en grandes áreas de terreno; 2) en el aire por los gases desprendidos, los incendios frecuentes, la generación de gas metano en grandes cantidades con su permanente olor a azufre, y 3) en las plagas de roedores y moscas que se generan permanentemente y que son vehículos de transmisión de muy diversas enfermedades.

Los métodos que han llegado a aplicarse en algunas cuantas ocasiones para frenar estos problemas, han sido parciales, deficientes o de muy corto alcance. Por ejemplo, existen algunas zonas en donde se practica un tipo (no siempre bien logrado) de relleno sanitario; hay también una planta industrializadora que no opera desde 1977; hubo tres incineradores que se construyeron en 1940 y que nunca pudieron funcionar, etc., y así se podrían mencionar diversos intentos para solucionar la problemática que se origina de la basura, pero la realidad rebasa por mucho las diferentes opciones que han tratado de implantarse en diferentes épocas.

Una parte importante de los procesos de recolección-disposición final de la basura, se dirige al rescate de los materiales reutilízables que son comercializados para distintos usos en los procesos industriales. Desde el momento en que la basura sale de manos de los consumidores (basura domiciliaria, hospitala ria y comercial) se inicia de hecho una primera selección, ya sea por parte de los barrenderos del DDF que pasan a recoger los desechos a las casas, o bien por parte de los empleados y voluntarios* de los camiones recolectores que reparten entre sí las ganancias obtenidas por la venta de materiales durante los recorridos de los vehículos, o también, como un caso extremo, por los pepenadores que deambulan por la ciudad recorriendo los tiraderos callejeros (llamados "clandestinos" por las autoridades) en la búsqueda de papel, botellas, cartón, fierro, etc. Aparte de esto habría que mencionar la basura de tipo industrial, sobre la cual no existe un registro específico y que, por lo general, llega directamente hacia los tiraderos sin ningún control (por parte del gobierno de la ciudad) sobre la peligrosidad o toxicidad que pueda contener. Ya en los tiraderos de basura, los desechos que llegan son

* Se conocen como "voluntarios" a las personas que viajan en los camiones de recolección de basura y que no siendo empleados directos del DDF, realizan labores de "pepena" (rescate) de materiales para que éstos sean vendidos en "depósitos particulares de desechos industriales" antes de llevar los desechos a las estaciones de transferencia o a los tiraderos. 
nuevamente "pepenados" ("nos llegan las puras migajas", comentan los pepenadores) separando, clasificando, empacando y almacenando la que será materia prima para muchas empresas, de lo que resulta un jugoso negocio en donde existe toda una compleja red de relaciones sociopolíticas y económicas que tienen sumidos en la miseria a más de dieciséis mil pepenadores, que son explotados por los concesionarios en su propio beneficio, obteniendo con ello cuantiosas ganancias, además de ejercer un absoluto y estricto control social sobre los trabajadores.

En la realidad, uno de los principales frenos que han existido para la interpretación de este problema es que tanto los factores técnicos (que están centrados básicamente en los procesos de recolección de basura, con casos aislados de aprovechamiento y de una racional disposición final), como los sociales (de la vida y organización interna de los tiraderos de basura a cielo abierto), se han analizado como dos aspectos desvinculados totalmente entre sí. Se carece, por parte de las autoridades, de una visión globalizadora (ecológica, económica, política y social) que permita desarrollar medidas directas que enfrenten el problema de la basura en la ciudad de México, y además, es un obstáculo digno de mencionarse, la existencia de relaciones informales entre los "encargados" públicos de la recolección y disposición final de los desechos con los líderes de los pepenadores, lo cual desemboca en un sistema corrupto, viciado, ineficiente y finalmente injusto para los pepenadores, que viven de trabajar con los desechos de la sociedad.

Para la ciudadanía, en general, el problema de la basura empieza en su casa y termina al depositarla en bolsas de plástico y entregarla al barrendero, o bien al tirarla en el camión recolector o simplemente al vaciarla en alguna esquina esperando que los empleados de Limpia y Transportes lleguen a recogerla. Sin embargo, es ahí donde justamente empieza el problema de la basura: ¿a dónde va?, ¿quién la compra?, ¿cuánto vale?, ¿para qué se usa?, ¿quiénes la venden a las empresas?, ¿cómo se depositan los desechos?, y luego, ¿cómo volvemos a consumir nuestra basura?

En el esquema 1 se presenta de manera gráfica el ciclo de circulación de desechos sólidos en el Distrito Federal, en donde pueden ser observadas las distintas etapas por las cuales van pasando los desechos desde su generación, recolección, hasta su reutilización por parte de la industria.

En lo referente a la recolección de basura, se trata de una actividad que, en la ciudad de México, desarrolla casi exclusivamente el Departamento del Distrito Federal (DDF), es decir, se trata de un servicio público. Puede estimarse que en la ciudad y su zona metropo- 


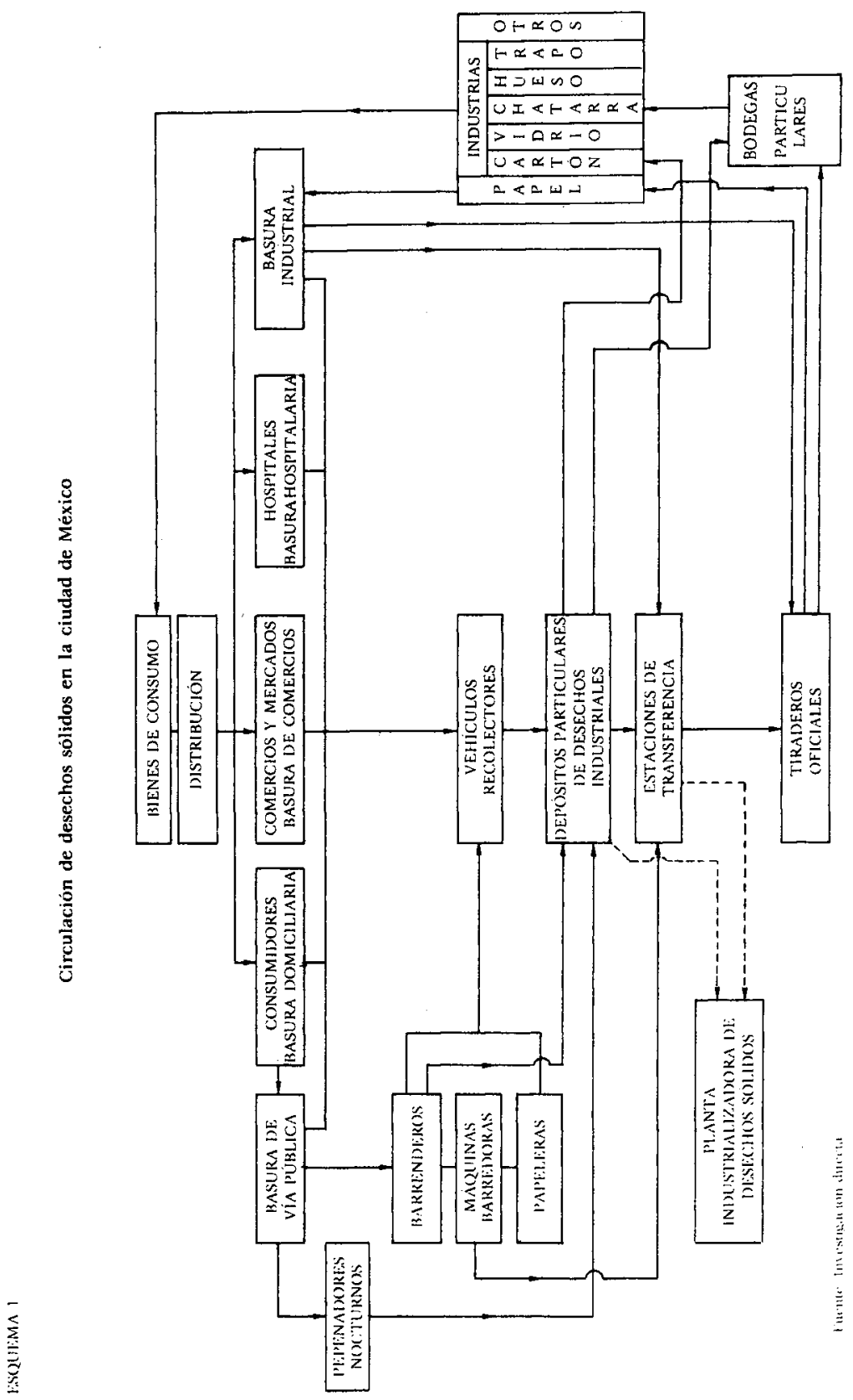


litana se generan diariamente entre 14000 y 16000 toneladas de basura. * Para su recolección se cuenta con aproximadamente tres mil quinientos vehículos recolectores (barredoras, volteos, compactadoras, trailers, etc.), sin embargo, una elevada proporción (35 a $40 \%$ ) no están en condiciones de operar normalmente. El servicio de limpia de la ciudad constituye una fuente de empleo para poco más de dieciséis mil trabajadores, que participan directa o indirectamente en la recolección de basura. El tipo de relaciones sociales que se generan hacia su interior se estructuran a partir de la existencia de verdaderos "feudos" tanto en las oficinas de Limpia de las delegaciones políticas como en las organizaciones sindicales existentes.

El servicio en sí mismo es deficiente: del total de toneladas producidas de basura sólo llega a recolectarse diariamente un promedio de $76 \%$, mientras que los desechos diarios por habitante fluctúan entre los 700 gramos y un kilo de basura.

Las delegaciones más céntricas de la ciudad (Gustavo A. Madero, Cuauhtémoc, Venustiano Carranza, Benito Juárez y Miguel Hidalgo) son las que producen más basura y en donde habita poco más de la mitad de la población del D.F.

Existen prácticas aceptadas consensualmente para repartir las ganancias diarias de las propinas entre barrenderos, "macheteros" (trabajadores que van en los camiones recolectores), voluntarios y choferes. Las formas de obtención de las mismas provienen de cuotas negociadas y cobradas a los distintos particulares y establecimientos comerciales, la venta a centenares de "depósitos particulares de desechos industriales" de los materiales pepenados arriba del camión, etcétera.

Quizá resultara injusto hablar de corrupción a este nivel, ya que la mayoría de los trabajadores de Limpia del DDF están sujetos al salario mínimo y las "ganancias extras" obtenidas por esta separación de materiales o bien por el servicio de recolección a los comercios, ${ }^{* *}$ apenas alcanza a ser un complemento adicional de este salario en la mayoría de los casos.

En el terreno de la disposición final debe señalarse que una vez que los trabajadores de los camiones recolectores ya pepenaron algunos materiales (botellas, fierro, papel, cartón, hueso, colchones, etc.) y los vendieron en los depósitos particulares, tienen dos opcio-

* Srgun estimaciones de la strut

** Esta practica se conoce en el medio como "tincluear", o sca recoger los desechos de las "fincas" que tiene cada ruta de camion recolector en sus diarios recorridos. 
nes: o van a una estación de transferencia en donde se llenan trailers (que evitan los viajes excesivos de los camiones a los tiraderos), o bien acuden directamente al tiradero que tengan asignado para depositar ahí lo que queda de la basura recolectada. En los tres principales tiraderos de la ciudad de México se encuentra la influencia de la principal organización de pepenadores del D.F., La Unión de Pepenadores de los Tiraderos del D.F. (UPTDF), agrupación formada a mediados de la década de los sesenta por el llamado "Zar de la Basura", Rafael Gutiérrez Moreno, quien fuera asesinado en marzo de 1987, y que pese a esto, la agrupación continúa con el control mayoritario de la basura de la ciudad.

En algunos tiraderos (Santa Catarina, Bordo de Xochiaca) su control es único y absoluto; en otros, (Santa Fe, parte baja) controla entre 60 y $80 \%$ de los terrenos y de los pepenadores y comparte el poder con otros "concesionarios" de menor tamaño. Los "viajes" de los camiones son repartidos entre las familias de pepenadores a través de los cabos* de la Unión de Pepenadores o bien de las otras organizaciones que existen en los tiraderos (Frente Único de Pepenadores de Santa Fe, A.C. y Comercializadora de Desechos Sólidos de Nezahualcóyotl, S.A. de C.V.).

El promedio de aprovechamiento de la basura en estas condiciones fluctúa entre 5 y $10 \%$ máximo del total que llega a los tiraderos. El precio promedio de la basura seleccionada que se compra a los pepenadores es de 21.00 pesos el kilogramo, mientras que el precio promedio de los materiales que son vendidos a las industrias es de 60.00 pesos el kilogramo.

Entre los principales problemas que pueden observarse al interior de los tiraderos de basura (en donde también viven los pepenadores) están: elevados grados de alcoholismo, drogadicción juvenil, desnutrición, altos índices de mortalidad infantil, insalubridad general en el trabajo, violencia y represión contra aquellos que se inconforman con los dirigentes del tiradero, y una dócil y bien aleccionada forma de apoyo incondicional a los líderes con el fin de que no se les quite la basura. Esto es, existe la idea de que si no protegen al tiradero y a sus organizaciones y dirigentes, "personas ajenas" que llegan a los tiraderos, podrían quitarles lo que hasta ahora es su único medio de sustento. Vale la pena mencionar que se estima que en los tiraderos de la ciudad de México $50 \%$ de pepenadores corresponde a mujeres, $25 \%$ a niños, $15 \%$ son hombres adultos y $10 \%$ son inválidos y ancianos, de los que más de $80 \%$ son pepena-

\footnotetext{
* Son los encargados de repartir los "viajes de basura" y de llevar el control de las familias que pueden pepenar en los tiraderos.
} 
dores nacidos en los mismos tiraderos e hijos de padres pepenadores. (Castillo Berthier, 1987, pp. 10-23).

Al mismo tiempo, en los tiraderos se generan una serie de relaciones sociales a partir de las cuales los intereses económicos de los líderes y concesionarios particulares encuentran protección política, a cambio del apoyo partidista al PRI en elecciones, mítines, etc., y de los acuerdos que establecen directamente con los Regentes de la ciudad.

En los tiraderos de basura de la República la "concesión" para explotar la basura, debe ser otorgada por las autoridades municipales a un "concesionario" (que puede ser privado o público), o bien, a alguna organización de trabajadores como es el caso que se verá a continuación, de los pepenadores de Ciudad Juárez, Chihuahua, que tienen establecida una cooperativa de seleccionadores. La complejidad de los procesos económicos y sociales que se generan a partir de estas actividades merece colocar nuevos interrogantes: ¿cómo se concesiona la explotación de la basura (y con ella la de los pepenadores) en los tiraderos de la ciudad de México?, ¿hacia dónde van las ganancias que reporta la venta de materiales a las industrias? Esto resulta importante en el momento actual, ya que si no se empieza por regular el concesionamiento de estas actividades* y por esclarecer las formas de operación del actual sistema de recolección-disposición final de los desechos, no se podrá avanzar en la solución del grave problema que representa, entre otras cosas, la creciente producción de basura de la capital, principalmente por la presión que ejercen las organizaciones de pepenadores para evitar que se les "quite" la basura y la imposibilidad ante esto de incorporar nuevos procesos menos contaminantes de tratamiento de desechos, por parte de las autoridades.

\subsection{El caso de Ciudad Juárez, Chihuahua}

En Ciudad Juárez, Chihuahua, los procesos de recolección y aprovechamiento de la basura presentan una racionalidad que supera las características de la disposición final de los desechos que se realiza, en general, en el resto del país y muy particularmente en la ciudad de México. Ello ha sido posible, entre otras cosas, por la existencia de una organización excepcional de los "trabajadores-seleccionadores" de ba-

* Debe mencionarse que en la ciudad de México, el concesionamiento para la explotación de los tiraderos se da totalmente de manera informal y no existe ninguna regulación o reglamentación formal al respecto. 
sura, la Sociedad Cooperativa de Seleccionadores de Materiales (soCOSEMA), y también porque las autoridades municipales del Departamento de Limpia prestan un servicio aceptablemente eficiente tanto en la recolección como en la aplicación de técnicas de semirelleno sanitario, resolviendo en buena parte los problemas normales que acarrean los tiraderos a cielo abierto.

Los diversos factores que coinciden en Ciudad Juárez, ofrecen una de las experiencias más interesantes en lo que respecta a la disposición de los desechos en el país. Se trata de una opción que articula la aplicación de técnicas susceptibles de ser empleadas para atenuar los efectos contaminantes de la basura sobre el medio ambiente de la ciudad, con una organización que permite a los trabajadores apropiarse del producto de su trabajo y mejorar así sus condiciones de vida y las del medio de trabajo en particular.

A diferencia del resto de los tiraderos del país, la disposición final de los desechos y su aprovechamiento, en este caso, permiten una distribución más justa de los ingresos y una organización del trabajo eficiente. Además, el ejercicio de una gestión colectiva ha eliminado la existencia anterior de formas tradicionales de caciquismo que, por lo general, prevalecen en esta clase de actividades.

Socosem es el ejemplo vivo de una de las alternativas que sugieren la posibilidad de implementar mejores tormas para el aprovechamiento de la basura como materia prima, al mismo tiempo que ofrece la posibilidad de que los grupos organizados de pepenadores contribuyan directa e indirectamente a modificar los efectos ambientales nocivos que provoca una inadecuada disposición final de los desechos, dado el control y manejo que pueden llegar a ejercer sobre la totalidad de los materiales que se depositan en tiradero, para realizar los procesos de beneficio y selección de la basura, y los sistemas técnicos que se pueden implementar para una mejor disposición final de los residuos que no son o no pueden ser aprovechados.

Las condiciones de trabajo que prevalecían en el tiradero de Ciudad Juárez antes de la constitución de SOCOSEMA en 1975, eran extremadamente desfavorables para los pepenadores, los cuales percibían bajísimas remuneraciones y soportaban pésimas condiciones de trabajo y de vida. En contrapartida, un concesionario particular estaba en condiciones de obtener importantes ganancias de la venta de los materiales seleccionados por los pepenadores. El 16 de mayo de 1975, adquirió personalidad jurídica esta organización, de la cual cabe mencionar todavía es única en el país. Para ello se obtuvo una concesión municipal y un permiso gubernamental especial que politizó inmediatamente esta actividad.

Los trabajadores supieron conseguir el apoyo del gobierno mu- 
nicipal y estatal para constituirse en cooperativa y obtener posteriormente la concesión que les permitiría usufructuar la basura que llega al tiradero, a fin de explotarla racionalmente para los intereses de la colectividad. Las posibilidades reales de que los trabajadores de la basura lograran estos objetivos descansaron en dos elementos fundamentales: a) poseer capacidad organizativa propia para constituirse en cooperativa de producción, y b) contar con la concesión que otorgó el municipio de Ciudad Juárez a esta empresa social.

El desarrollo de la cooperativa pudo afianzarse gracias a la firma del contrato-concesión que formalizaba el compromiso por el cual las autoridades municipales entregaban a socosema la explotación común del basurero municipal e igualmente el uso de los permisos de internación de desechos provenientes de otros tiraderos de la frontera sur de los Estados Unidos. La contrapartida, que actualmente favorece directamente los intereses de los cooperativistas, era la de aportar a la Hacienda Pública Municipal, la cantidad de \$75 000.00 mensuales, como contraprestación a la concesión otorgada. Esta cláusula está vigente aún sin modificaciones.

La sociedad cooperativa contó con la asistencia de un grupo de asesores que apoyó las intenciones de los agremiados durante varios años, mientras adquirían la capacidad para resolver autónomamente los diversos aspectos legales y políticos, sociales y económicos que requiere el funcionamiento de una empresa social que, como ésta, entrenta sistemáticamente poderosos intereses.

Al igual que cualquier otra cooperativa, socosema, formada por un grupo de origen popular, tuvo que enfrentar un conjunto de obstáculos para consolidarse. Entre éstos se pueden señalar los siguientes:

Obstáculos económicos. El principal problema que afrontó en el inicio, fue el continuar percibiendo un ingreso monetario estable durante el periodo en el que dejaron de trabajar para el concesionario. También hubo que conseguir la suma indicada por el municipio como pago por la concesión. En ambos casos, y al parecer también en otros momentos económicos difíciles por los que atravesó la organización, contaron con el apoyo de un importante empresario local que ayudó al financiamiento de estas etapas. Por otra parte, mejorar las condiciones económicas implicaba no sólo garantizar una mejor remuneración por el trabajo realizado sino modificar el conjunto de condiciones de vida de estas familias. En lo fundamental, lo inmediato fue concientizarlos de que su lugar de habitación debería estar fuera del tiradero. 
Obstáculos sociales. Podemos incluir aquí los provenientes de la misma condición social de los pepenadores. El tratarse de un sector de trabajadores urbanos que percibe escasos ingresos, posee un nivel educacional bajo y condiciones de vida de extrema pobreza fue el móvil por el cual el grupo de asesores decidió ayudar a constituir la cooperativa. Sin embargo, en estas mismas características radican las limitaciones para que se desarrolle un proceso de autogestión colectivo, pues durante muchos años existieron fuertes mecanismos de dependencia con los asesores y agentes externos que participaron en la administración inicial de la cooperativa.

Obstáculos políticos. Inicialmente, los trabajadores del tiradero de Ciudad Juárez pertenecían a la Corporación Revolucionaria Obrera y Campesina (CROC) y a la Liga Municipal de Organizaciones Populares (LMOP). Ambas son organizaciones ligadas al Partido Revolucionario Institucional, cuyos representantes exigian a los pepenadores el pago de una cuota mensual, además de usarlos como grupos de apoyo en mítines y campañas políticas. La posibilidad de formar una cooperativa autónoma debió enfrentar los intereses políticos y económicos de los dirigentes locales. Un apoyo político fundamental fue el lograr interesar y comprometer al presidente municipal, aunque esto requirió de una campaña de presión bien organizada a través de la prensa local para comprometer públicamente al presidente en favor de la cooperativa.

Obstáculos culturales. Son los que están ligados a la forma como los sectores populares deben vencer unà serie de resistencias para enfrentar los requisitos que imponen prácticas tales como realizar los trámites burocráticos en los organismos gubernamentales, la defensa de los derechos que les corresponden, la posibilidad de crear formas de producción basadas en la cooperación y en la igualdad, el aprender conocimientos técnicos para la gestión, etcétera.

Estos obstáculos se fueron resolviendo paulatinamente, y dieron lugar a nuevos retos, muchos de los cuales animan actualmente a la cooperativa. De los 224 socios iniciales, contaba con 210 , en 1985 (según su nómina de salario mensual) y con un número poco menor de trabajadores eventuales conocidos como "extras". Entre los beneficios económicos y sociales de que disfrutan los socios destacan la estabilidad y seguridad en el empleo, que para este sector social constituye un aspecto muy importante debido a que para una mano de obra no calificada, una de las barreras que le impone el mercado de trabajo es el obtener una incorporación regular a la actividad productiva.

En resumen, los cooperativistas de socosema cuentan ya con un nivel de remuneración adecuado y gozan de estabilidad en el traba- 
jo. A ello se agrega el acceso a diversos servicios sociales fundamentales: alfabetización para sus asociados, atención médica para el trabajador y su familia, y un programa de vivienda fuera del tiradero de basura, entre otros.

Por otra parte, debe mencionarse que los aspectos técnicos referentes a la recolección de basura están a cargo del municipio, con cabecera en la misma Ciudad Juárez. Se trata de un servicio público que se complementa de manera poco significativa con los servicios periódicos de recolección de los desperdicios de maquiladoras, que se realizan por cuenta de algunos miembros de la cooperativa.

En la ciudad se generan alrededor de seiscientas toneladas diarias de basura y se cuenta con número considerable de vehículos recolectores en operación (barredoras, volteos, compactadoras y otros vehículos). Para el Departamento de Limpia trabajan directamente alrededor de ciento veinte personas y en el proceso global de recolección y aprovechamiento se estima que llegan a participar alrededor de tres mil personas diariamente, a muy distintos niveles.

Cada día se recolecta alrededor de $80 \%$ del total de toneladas de basura producidas en la ciudad, en tanto que el promedio de desechos diarios por habitante fluctúa entre 500 y 750 gramos $(1.5 \mathrm{mi}$ llones de habitantes).

El tiradero de Ciudad Juárez tiene una extensión de 100 hectáreas; se sitúa al sureste de la ciudad en una formación montañosa con suelo calizo y tierras pobres. El terreno es irregular, cuenta con hondonadas pronunciadas y barrancos que se aprovechan para el semirrelleno sanitario con que se ha venido resolviendo el problema de la basura a cielo abierto. Aunque de hecho no se practica un relleno sanitario con todas las normas específicas que se requieren, el sistema que se utiliza permite cubrir con una capa delgada de tierra los desechos que se depositan en los barrancos y hondonadas cada 24 horas, con lo cual se limita gran parte de la contaminación en aire, suelo y en la proliferación de fauna nociva.

Las autoridades municipales se han encargado de establecer el lugar del tiradero, mismo que han modificado en varias ocasiones. No se cuenta con ninguna planta industrializadora de desechos aunque se ha estudiado la factibilidad de introducirla por parte de la cooperativa. En enero de 1985 se estimaba su costo en 300 millones de pesos.

En el volumen de los materiales rescatados, el cartón constituye el material más importante; representa más de la mitad de los ingresos que obtiene la cooperativa por venta de materiales. Le siguen en importancia el papel y el cartón maquila, que constituyen las otras principales fuentes de ingreso. 
La venta de productos introduce a la cooperativa en u complejo marco de relaciones comerciales que van desde la determinación de los precios, búsqueda de mercados, atención a los clientes y programación de la producción hasta la distribución, promoción de sus productos, distribución interna de ganancias y otros aspectos administrativos.

La venta de productos es el resultado formal del proceso de selección de los desechos para su disposición final; sin embargo, otro tipo de aspectos como sería la identificación grupal para enfrentar los problemas de la ciudad, es decir, el reconocimiento de los miembros de la cooperativa como parte de la población y de la vida de Ciudad Juárez, ha sido fundamental para legitimar y vitalizar la existencia de la cooperativa.

La venta de productos, y por ende su control, otorga reconocimiento sobre la utilidad social de su trabajo y es a la vez un medio de identificación y valoración de su actividad. A través de ello, el manejo autónomo de la venta de productos les ha permitido consolidar la operación de la cooperativa y explorar diversas maneras de coordinación y división del trabajo, así como iniciar una revisión detenida de las perspectivas que tiene la selección de la basura. Tal sería el caso de los proyectos de formación de subsidiarias de la soCOSEMA, o la extensión del proceso hacia otros tiraderos del norte del país, la construcción de una planta industrializadora, el aumento de sueldos, y la mejor atención a las condiciones de habitación, salud, educación e integración comunitaria y social.

La venta de productos ha podido significar lo anterior porque la cooperativa tiene autonomía política, autosuficiencia financiera $e$ independencia administrativa para organizarse y tomar las decisiones relacionadas con todas sus actividades. Esto quiere decir que cuenta con el poder suficiente para negociar directamente con los clientes, la competencia, las autoridades municipales o bien los bancos. Por esto pueden discernir con claridad cómo encontrar los mejores términos de sus distintas operaciones.

La venta de productos y el contacto con el mercado les ha llevado a aplicar criterios para aprovechar sólo determinados productos $\mathrm{y}$, a la vez, les ha permitido pensar en la cantidad y diversidad de otros materiales que también podrían procesar. De lo primero es evidente la lista de productos reunidos; de lo segundo es el trapo, hueso, madera y los proyectos de adquisición de maquinarias para triturar y moler plástico y vidrio e iniciar un proceso de semi-industrialización que les redituaría, desde luego, mayores ingresos. Ello indica que la cooperativa ha empezado a superar las condiciones técnicas con que opera y requiere transformar las caracte- 
rísticas del proceso de aprovechamiento de los materiales y con ello su organización para la producción.

El ingreso promedio semanal por la venta de cartón, bote, papel, "cartón maquila", hule, arpillas, aluminio, estibas, pacas, botella comercial y sueldos de raya era en 1984, de casi un millón quienientos mil pesos. Por su parte, el precio de venta promedio de los materiales para los cuales se dispone de precio estable era en enero de 1985 de 10.92 pesos el kilogramo. El ingreso promedio semanal mencionado equivale a un volumen de materiales aprovechados de 128558.33 kilogramos. Se considera que en promedio sólo se aprovecha $5 \%$ del volumen total de la basura y que con apoyo para desarrollar tecnologías intermedias de fácil construcción su aprovechamiento potencial sería de hasta 25 por ciento.

Cada una de las 270 personas que trabajan simultáneamente, manipula alrededor de 1.85 toneladas diariamente, de las cuales extraen un volumen de $92.4 \mathrm{~kg}$ equivalente en 1984 a 1009.92 pesos en promedio. Puede afirmarse entonces que existen posibilidades de incrementos, tanto en el porcentaje de materiales aprovechados como en los ingresos, en por lo menos dos direcciones: una cuantitativa y otra selectiva.

Según los datos examinados se puede concluir que los trabajadores alcanzan un ingreso comparable con el minimo vigente y que pueden rebasarlo hasta en $35 \%$. También se observa que la jornada promedio de trabajo es de 6 horas como máximo, lo cual es un límite necesario para este tipo de actividad. Lo fundamental entonces es que esta experiencia, aunque propia de un determinado espacio urbano y en condiciones socioeconómicas y políticas particulares, permite pensar en la existencia de formas técnicas y sociales alternativas que legitimen y reconozcan la función social productiva de los pepenadores; que incrementen la recuperación de materiales disminuyendo paralelamente los volúmenes de desechos, y que, finalmente, puedan ser objeto de una racional planificación para la incorporación de tecnologías simples que reduzcan a su mínima expresión, los impactos nocivos al medio ambiente.

3. Principales técnicas para el control de la contaminación de desechos sólidos

Sin querer profundizar exhaustivamente sobre los distintos aspectos que se presentan en la contaminación ambiental (fuentes fijas, móviles, elementos tóxicos, carga orgánica, etc.), se presentan a continuación algunas características sobre la contaminación (principalmente en el suelo y en menor medida en el aire y agua) por resi- 
duos sólidos que son observables en México, así como las alternativas que se han planteado para combatir este problema.

En términos generales los desechos sólidos pueden dividirse en dos grandes grupos: 1) aquéllos identificables como no putrecibles o no biodegradables (vidrio, lámina, plásticos, etc.), y 2) aquéllos comprendidos dentro de la carga orgánica que sí son biodegradables y que pueden incorporarse nuevamente, casi de manera natural, al medio ambiente.

El primer grupo de productos representa un promedio de casi $50 \%$ de los desechos que se generaban en la ciudad de México en $1976 .{ }^{8}$ Sin embargo, debe señalarse que los desechos no biodegradables se van incrementando conforme avanza el consumo de productos enlatados o empaquetados. Esta situación puede verificarse en otras sociedades con mayor grado de industrialización, como Japón o Estados Unidos, donde los desechos no orgánicos ocupan ya los mayores porcentajes de desperdicios. Es decir, existe correspondencia entre el grado de desarrollo industrial de una sociedad y el nivel y composición de desechos de la misma, los cuales son muy similares en casi todas las ciudades de América Latina.

En el caso de México los desechos no biodegradables generan las actividades de "pepena". Es decir, se trata de un proceso de selección y clasificación que permite reaprovechar entre $5 \%$ y $10 \%$ del total de los desechos en diversos procesos industriales. Como se ha visto, estas actividades encierran una compleja problemática económica, política y social particular.

En cuanto a los desechos orgánicos, que representan otro $50 \%$ de los desechos totales, son poco utilizados quedando esparcidos en grandes áreas de disposición final (tiraderos a cielo abierto), que son fuentes permanentes de contaminación ambiental.

Dentro de las alternativas tecnológicas que se han propuesto para resolver (aunque sea parcialmente) el problema de la contaminación por desechos sólidos en México, están las siguientes:

\section{a) Incineración}

Una vez realizada la pepena o rescate de los principales materiales que son reciclables, se procede a quemarla. La materia orgánica, el oxígeno y el calor producen bióxido de carbono, agua y gases que son elementos contaminantes, y que para poder ser arrojados al medio ambiente deben pasar por algún proceso de purificación del aire (ciclones, bolsas o precipitadores electrostáticos, etc.), lo cual trans-

${ }^{8}$ Datos de la extinta Dirección General de Servicios Urbanos del DDF. 
forma el método de incineración en un proceso sumamente costoso que no resuelve, sino parcialmente, el problema. En algunos casos se considera que es posible la transformación del calor generado en energía eléctrica. Sin embargo, esto requiere de una muy sofisticada tecnología que no se produce en el país y que está fuera de las posibilidades económicas actuales de México.

Pese a esto, en la ciudad de México se está terminando de instalar el primer incinerador de basura en lo que fuera la PIDS (Planta Industrializadora de Desechos Sólidos) de San Juan de Aragón, que tiene una capacidad para incinerar 500 ton al día, que representan a lo sumo $3 \%$ de la basura total de la capital mexicana.

\section{b) Reciclaje-composta}

Este proceso aerobio fue el que se trató de instalar originalmente en la PIDS; consiste en la recuperación manual de materiales reciclables para aislar la materia orgánica que posteriormente se transforma en "composta", utilizable como mejorador de suelos; el residuo o rechazo se aplica a un relleno sanitario. La composta se obtiene al separar la materia orgánica y apilarla horizontalmente dejando que las bacterias aeróbicas vayan alimentándose de los desechos. Estas pilas deben ser removidas cada semana para dar entrada al oxígeno y a la humedad, con lo cual se obtiene bióxido de carbono, agua y un "humus" que es rico en fósforo y nitrógeno y que puede ser utilizado como fertilizante. Este proceso aerobio tiene una duración de seis meses en promedio, por lo cual es dificil imaginar las enormes dimensiones de terreno que se necesitarían para ir aprovechando la basura de la ciudad de México y su zona metropolitana (14 000 ton / día). Sin embargo, este mismo proceso puede ser acelerado por los llamados digestores (tubulares o verticales) que lo reducen entre 48 y 72 horas para obtener la composta, y que también son parte de una costosa tecnología que México no produce.

\section{c) Pirólisis}

Este es un proceso anaerobio del cual se pueden obtener algunos hidrocarburos. Consiste en separar la materia orgánica aplicándole calor directamente pero sin permitir la entrada de oxígeno, con lo cual se le puede transformar en gas metano, carbón y petróleo. Sin embargo, siendo éste uno de los procesos más costosos y lentos, está por demás decir que se ubica totalmente fuera de las posibilidades económicas del país. 


\section{d) Relleno sanitario}

Este es el proceso más barato de todos los enunciados. Se encuentra en una situación de 10 a 1 en lo que a costos se refiere para cualquiera de las otras tres alternativas.

El relleno sanitario consiste primero en rescatar los materiales inorgánicos reciclables para después apilar los desechos restantes en montañas de basura qu son cubiertas con capas de 70 centímetros de tierra, pudiendo volver a depositar nuevamente más basura orgánica encima de ellas. Asimismo, en las áreas de relleno sanitario, se pueden instalar "torres" construidas en forma de cubos de fierro y alambre, con piedras en el interior para que se permita la salida de los gases que se van produciendo al interior de la materia orgánica, con el fin de prevenir los frecuentes incendios. Esto es, la materia orgánica entra en proceso de putrefacción alcanzando muy altas temperaturas que a su vez generan bacterias anaerobias que se van alimentando de los desechos y que llegan a producir gas metano, bióxido de carbono y nitrógeno.

A pesar de ser un proceso lento, es el que mayor volumen de basura puede recibir. Después de 8 meses (en promedio), puede empezarse a obtener gas metano, que puede reutilizarse como combustible. Una experiencia exitosa de este proceso se halla en la ciudad de Santiago de Chile, en donde a través del relleno sanitario ya se está cubriendo un alto porcentaje de las necesidades de gas para las estufas de la población.

A través de este método se puede regular asimismo la contaminación de los mantos acuíferos y los problemas de las moscas y los roedores que se vuelven totalmente incontrolables en los tiraderos a cielo abierto, como es el caso de la ciudad de México.

Por esto, tal parece que en el momento actual la técnica de relleno sanitario parece ser el medio de control más barato y eficaz para frenar la contaminación ambiental que producen los desechos sólidos, dado que no elimina de entrada el trabajo de selección de materiales por parte de los pepenadores, además de que requiere de una inversión mínima de capital para su funcionamiento, en comparación con otras técnicas.

\section{Conclusiones y recomendaciones}

Se ha presentado hasta aquí una breve panorámica de las principales características que pueden encontrarse en los tiraderos de basura de la ciudad de México y Ciudad Juárez, Chihuahua, así como de las técnicas de disposición final más difundidas, evaluando cuál 
de estas últimas se ajusta más a las necesidades de nuestra realidad actual.

Es un hecho palpable que hay efectos ambientales nocivos por la existencia de tiraderos a cielo abierto, y que también se ha tratado de frenar el crecimiento de estos últimos con diferentes medidas que se han aplicado en distintas épocas, sin embargo ¿qué ha pasado?, ¿por qué no han podido establecerse nuevos sistemas de disposición final de los desechos?

Uno de los primeros intentos se dio en 1941, cuando se instalaron tres plantas incineradoras de basura, una en el aeropuerto, otra en Azcapotzalco y otra más en San Andrés que, a decir de algunos empleados del DDF en esa época, "operaban con tecnología y empleados extranjeros". Al parecer, la instalación de estas plantas propició inmediatamente la inconformidad del principal concesionario de los tiraderos en aquel entonces, quien temía que su fuente de ingresos disminuyera o al menos se viera seriamente afectada y, curiosamente apuntan, "en ese mismo año las plantas dejaron de funcionar después de sufrir incendios simultáneamente, presumiéndose que estos hechos fueron planeados por él mismo". (Castillo Berthier, 1987).

Otro intento más reciente fue la instalación de la Planta Industrializadora de Desechos Sólidos de San Juan de Aragón, ciudad de México, en 1974, en la cual se procesarian diariamente cerca de setecientas toneladas de basura para obtener composta. En dicha planta fueron incorporados 40 pepenadores del antiguo tiradero de Santa Cruz Meyehualco, para realizar labores de selección de materiales antes de tratar la materia orgánica; sin embargo, en la primera semana de trabajo 38 de estos trabajadores regresaron al tiradero y los dos restantes lo hicieron días después, ya que no estaban dispuestos o al menos acostumbrados a una rutina burocrática que substituyera el trabajo a destajo que realizaban diariamente y tampoco a usar el equipo de trabajo que se les proporcionó (uniforme, guantes, mascarilla, casco, etc.), con lo cual, aunado a la mala administración y a las frecuentes descomposturas del equipo sueco que se importó para su instalación y la falta de refacciones para la maquinaria, quedó inutilizada desde principios de 1977, hasta que hace un par de años se instaló un gigantesco incinerador alemán que todavía no se pone a funcionar.

En 1982, con la clausura de Santa Cruz Meyehualco, se anunció el relleno sanitario del área que ocupara este tiradero, para plantar docenas de miles de árboles que formarian posteriormente un parque recreativo. Sin embargo, durante el primer mes de siembra murieron más de $95 \%$ de los árboles plantados, dejando sin ningún uso específico las más de 160 hectáreas de "tierra muerta" que conforman hoy este lugar. 
Y así, quizá fuera posible seguir dando ejemplos que expliquen el fracaso de las opciones técnicas que se han dado para afrontar el problema de los desechos sólidos, de los cuales, muy probablemente, se podría obtener la conclusión de que en forma muy directa han sido los concesionarios, $\mathrm{y} / \mathrm{o}$ los pepenadores, a través de sus líderes y organizaciones gremiales, quienes mayor resistencia y oposición han mostrado a la incorporación de estos intentos.

Por ello, hablar de los impactos ambientales que produce la basura en el medio urbano, obliga forzosamente a remitirse al análisis sociológico de los pepenadores no solamente como grupo marginal, ni tampoco como un gremio explotado por líderes corruptos, sino más bien como un sector que es el verdadero "dueño" de la basura de la sociedad, posición que ha ganado por las muchas décadas que tiene de trabajar con los despojos de la ciudad, encontrando en ellos un medio de vida y de supervivencia para miles de personas que habitan en los tiraderos.

No entender esto sería no entender la verdadera magnitud del problema de la basura en México y, un poco más allá del mero entendimiento, cualquier plan o acción que se aboque a la solución técnica del problema de los desechos sólidos, sin considerar la parte social que existe en los tiraderos, terminará, al menos en las condiciones actuales, siendo un fracaso.

Por todo esto, este pequeño trabajo intenta plantear algunas consideraciones necesarias para entender en forma global los problemas que acarrea la generación y el destino final de los desechos urbanos:

1) La basura, sea ésta del tipo que sea (domiciliaria, comercial, hospitalaria, de mercados, industrial, etc.), llega en un alto porcentaje directamente a los tiraderos a cielo abierto ya que no existe actualmente un control específico para tratar distintos tipos de basura.

2) La basura, antes de ser desecho, es un negocio que reporta altas utilidades para unos cuantos y da empleo a miles de pepenadores que trabajan en los tiraderos.

3) Existe un mercado en ascenso que demanda los distintos materiales que pueden obtenerse de la basura.

4) La generación creciente de basura inorgánica obliga a pensar en nuevas formas para aprovechar mayores porcentajes de desperdicios para usos industriales.

5) Las organizaciones de pepenadores limitan las posibilidades de introducir nuevas tecnologías para tratar los desechos, si no se les respeta su actual fuente de empleo.

6) Por ello, una respuesta "social" para dar formalmente la categoría de trabajadores a los actuales pepenadores, a través de la orga- 
nización de cooperativas de producción o agrupaciones similares, permitiría ir planificando el futuro de los tiraderos para que dejaran de ser lugares contaminantes, sitios de vivienda precaria, etc., para que se transformaran en auténticas empresas que dieran valor a los desechos evitando, a la vez, el crecimiento indiscriminado de los pepenadores.

A pesar de que la idea de reorganizar los procesos de trabajo en los tiraderos parecería ser una posible solución para iniciar un proceso de racionalización sobre el destino final de los desechos sólidos, en el caso de la ciudad de México, esto es particularmente difícil dados los intereses creados que existen alrededor de este negocio, y entre los cuales valdría la pena resaltar los siguientes:

a) De los tiraderos existentes, el de Santa Catarina es practicamente impenetrable ya que desde su entrada, guardias fuertemente armados custodian el lugar para evitar la entrada a personas "ajenas" al negocio que ahí se desarrolla. Situación que es similar en la parte baja de Santa Fe (actualmente ubicada en Prados de la Montaña) y en Xochiaca, que tradicionalmente han sido controlados por un mismo concesionario.

b) Los pepenadores que trabajan en los tiraderos de la ciudad, en general, habitan en los mismos sitios de trabajo en condiciones insalubres y a veces infrahumanas, además de que no reciben en todos los casos una remuneración equitativa por su trabajo, dejando las ganancias que se obtienen de la separación de materiales en manos de terceros que sólo fungen como intermediários.

c) Las actuales organizaciones de pepenadores sirven para disfrazar la verdadera explotación a que se somete a los pepenadores bajo una apariencia de organización sindical que, en la realidad, sólo es manipulada en beneficio de los intereses particulares de sus líderes y dirigentes.

d) A pesar de que la función que cumplen estos líderes es la de ser los concesionarios directos de la basura, no existe ninguna reglamentación oficial al respecto ni tampoco, a simple vista, ninguna limitación para ejercer su dominio abierta y directamente de acuerdo a sus intereses particulares.

e) Por su parte, las autoridades se muestran reacias a reconocer abiertamente este problema: la SEDUE dice no poder tener injerencia alguna ya que es un problema que atañe única y exclusivamente al DDF, mientras que en el DDF se niega cualquier información a este respecto y no se autorizan visitas a los tiraderos aduciendo que "son lugares que resultan muy peligrosos" y que no pueden hacerse responsables de lo que ocurra "allá adentro".

f) A su vez, los concesionarios aseguran que los tiraderos son 
considerados como "propiedad privada" y que es por eso que se niega la entrada, a pesar de que ocupan normalmente terrenos de propiedad federal.

g) Los concesionarios de los tiraderos cumplen una función económica importante al convertirse en abastecedores directos de diversas empresas que reciclan materiales de desecho.

h) Los concesionarios también juegan un papel político importante al convertirse en líderes de sus organizaciones populares, las cuales apoyan y, al mismo tiempo, se respaldan en el gobierno a través de su pertenencia al partido oficial y a las organizaciones que de él dependen, participando como grupos de "acarreados" en mítines, campañas políticas y en el momento de las elecciones.

i) Asimismo, el Estado legitima el poder de los concesionarios al reconocerlos como líderes de sus comunidades y al transformarlos en el principal "canal de beneficios" (agua, luz, tiendas con alimentos subsidiados, etc.) para los pepenadores, aparte de que en algunos casos han llegado a tener diversos cargos públicos (diputados, jefes de relleno sanitario, etcétera).

j) Por otro lado existe la advertencia de los líderes hacia los pepenadores de que no deben permitir la entrada a nadie extraño a los tiraderos porque "lo que buscan todos es apropiarse de la basura", ya sea para industrializarla o simplemente "enterrarla" con lo cual les quitarian su única fuente de ingresos, transformándose esta amenaza en uno de los principales impedimentos para buscar alguna otra solución. "La tierra es de quien la trabaja, la basura es de los pepenadores que la trabajan", es una de las frases cotidianas que utilizan los líderes de los pepenadores para advertir esta situación.

Por todo esto, tanto la existencia de los concesionarios como su permanente comunión entre ellos y el Estado, hacen que resulte difícil poder vislumbrar alguna propuesta de cambio que altere estas aparentes buenas relaciones de clientelismo político que se tienen.

Existen además muchos otros problemas que van corriendo paralelamente a los ya señalados y que van desde el tiem po político sexenal y las remociones de funcionarios que ello trae consigo, y la voluntad específica que necesita un regente de la ciudad para hacer frente al problema.

Dado que los concesionarios de los tiraderos en la ciudad tienen y han tenido a lo largo de su historia el acceso directo con los regent de la ciudad para negociar privadamente la forma de manejo de ins tiraderos, quizá fuera necesario encontrar la buena voluntad de algún jefe del Departamento para acordar con los concesionarios la forma de implementar más organizadamente los rellenos sanitarios, incorporar tecnologías intermedias que permitan aumen- 
tar los porcentajes de aprovechamiento de materiales y quizá llegar a sacar las viviendas de los pepenadores de los tiraderos, ya que es este último elemento un factor de peso para la consolidación de la fuerza de trabajo (no se olvide que poco más de $80 \%$ de los pepenadores han nacido ahí y son hijos de padres pepenadores), creando un círculo vicioso en donde los niños que nacen en el tiradero no llegan a tener otra visión del mundo, dado que se incorporan al trabajo familiar desde muy temprana edad. En contraposición a esto, debe mencionarse el caso de Ciudad Juárez, en donde a 10 años de la fundación de la cooperativa y de haber puesto las viviendas de los trabajadores fuera del tiradero, el número de pepenadores ha disminuido y la escolaridad aumentado, permitiendo encontrar mejores opciones de empleo para los familiares de los trabajadores.

La ciudad de México presenta un estudio de caso que merece un estudio particular de su problemática. Sin embargo, los impactos ambientales que provocan los desechos sólidos existen en mayor o menor escala en todos los tiraderos a cielo abierto que hay en todas las ciudades de la República. En muchas de ellas hay pepenadores, organizados o trabajando independientemente, lo cual obliga a ver el problema en una perspectiva macrosocial. Es decir, reconociendo que previamente a ser desecho la basura es antes que otra cosa un negocio y una alternativa de recuperar materias primas necesarias para distintos procesos industriales; que de ella viven miles de personas, y que su uso bien organizado puede dismunuir el consumo de recursos naturales renovables y no renovables, las alternativas para la organización de los procesos de trabajo resultan imprscindibles para contrarrestar los impactos ambientales que se tienen actualmente, al menos en dos niveles: el uso indiscriminado de recursos naturales y los efectos nocivos al medio ambiente urbano.

Quizá a través de estos incipientes trabajos sobre el tema pudiera integrarse una metodología de evaluación, ecológica y social, para desechos sólidos, aplicable no sólo a las ciudades de México sino también a muchas otras de América Latina, dadas las enormes similitudes y problemas que las afectan, con el fin de homogeneizar y solucionar globalmente un problema que surge en distintos lugares, pero con una misma racionalidad y con efectos similares. 


\section{Bibliografia}

Castillo Berthier, Héctor, "La sociedad de la basura: caciquismo en la ciudad de México", IISUNAM, Cuadernos de Investigación Social, núm. 9, México, D.F., 1983.

Castillo, Héctor, Camarena, Margarita y Ziccardi, Alicia, La sociedad cooperativa de segregadores de materiales de Ciudad Juárez, Chihuahua, (mimeo) SEDUE, 1985.

Castillo Berthier, Héctor, Reciclaje de Desechos en la ciudad de México: La Participación del Sector Informal de los Pepenadores, ilsunam, México, (mimeo), 1987.

Leff, Enrique, "Ecologia y capital una reflexión teórica", en Antropologia y Marxismo, núm. 3, abril/sept., 1980, pp. 67-75, México, D.F.

- Racionalidad, ecotecnologia y manejo integrado de recursos: hacia una sociedad ncguentrópica" en Revista Intcrancricana eic Plenificacion, vol. xv'll, num. 69, marzo, 1984, pp. 70-85.

Ojeda, Olga y Sánchez, Vicente, La cuestión ambiental y la articulación sociedadnaturaleza. Programa Desarrollo y Medio Ambiente, El Colegio de México, México, 1984, (Documento de trabajo).

Pires, Pedro, "Notas sobre politica regional y medio ambiente", en Cicneias Lrbeness, núm. 1, SAHOP, México, D.F., marzo, 1982, pp. 19-24.

Restrepo, Ivan y Phillips, David, La basura, consumo y desperdicio en el Distrito Federal, Instituto Nacional del Consumidor, México, D.F., 1982.

Sachs, Ignacy, Ecodesarrollo, desarrollo sin destrucción, El Colegio de México, México, 1982.

SAHOP, Dirección General de Desarrollo Ecológico de los Asentamientos Humanos, Estudio, recolección y disposición final de los desechos sólidos para Ciudad Juárez, Chihuahua, México, D.F., (mimeo), 1982.

Sánchez, Vicente, "Notas sobre medio ambiente y planificación del desarrollo" en Ciencias Urbanas, núm. 1, SAHOP, México, D.F., mayo, 1982, pp. 13-18

- La problemaitica del medio ambicnte y la planificacion. Programa de: Jesarrollo Y' Medio Ambicnte, El Colegio de: México, México, 1983, (Documento de Trabajo) - "Impacto ambiental de los ascontamientos humanos: notas conceptuales y me-

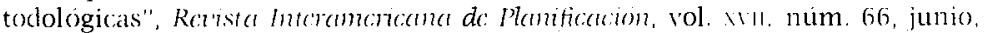
1983, pp. 175-184.

Secretaria de Programación y Presupuesto, Plan Nacional de Desarrollo 1983-1988, México, D.F., 1983.

- Fortalecimicnto y Desarollo Muncipal, num. 5. Mexico, 1).F., septicmbre de: 198.3.

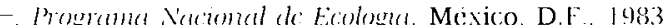

Trueba, José, Ecologíı para el pueblo, Edicol, México, D.F., 1980.

Willis, L.U. Estudio socioeconómico de la Sociedad Cooperativa sorosent. Ciudad Juárez, Chihuahua, 1980 (mimeo). 
